\title{
Extended spectrum $\beta$-Lactamase production and the Co-existed antibiotic resistance among Uropathogenic Escherichia coli isolates
}

\author{
Mona A. Al-sayed, Ashraf A. Kadry, Amira M. El-Ganiny, Nour M. Al-Kashef* \\ Microbiology and Immunology Department, Faculty of Pharmacy, Zagazig University, \\ Zagazig, Egypt.
}

*Corresponding author e-mail: nour_alkashef@yahoo.com

\begin{abstract}
Uropathogenic Escherichia coli is the main uropathogen involved in community and hospital acquired urinary tract infections. The increased rate of resistance towards different classes of antibiotics limits the treatment options for such infections. This study aim to determine the local resistance rate against different classes of antibiotics that are commonly used in treatment of urinary tract infections. Also to determine the rate of ESBL production and the correlation with other antibiotic resistance among producing isolates.

In this study, 112 UPEC isolates were collected from patients who were suffered from symptoms of UTIs, antibiotic susceptibility was determined by disk diffusion method against $\beta$-lactams, fluoro-quinolones, folate pathway inhibitors, aminoglycosides, tetracyclines and nitrofurans. Also, Extended spectrum $\beta$-lactamase (ESBL) production rate was determined based on the observed reduction in susceptibility towards $\beta$-lactams antibiotics in routine susceptibility testing which confirmed using double disk synergy test.

The UPEC isolates exhibited increased resistance rate towards different antibiotic classes especially sulphamethoxazole-trimethoprim, fluoro-quinolones and $\beta$-lactams which are mainly used in the treatment. About $50 \%$ of UPEC isolates were found to be ESBL producing. The ESBL producing isolates exhibited high resistance rate towards non $\beta$-lactam antibiotic especially sulphamethoxazole-trimethoprim and fluoro-quinolones $(67.8 \%$ and $63 \%$ respectively.) along with other antibiotics. However, ESBL producing isolates exhibited low resistance against piperacilin-tazobactam, nitrofurantoin, imipenem and meropenem $(17.8 \%$, $16 \%, 3.5 \%$ and $1.7 \%$, respectively). Therefore, it is recommended to use piperacilintazobactam, nitrofurantoin, imipenem or meropenem in treatment of UPEC resistant strains
\end{abstract}

Key words: Urinary tract infections, UPEC, ESBL, multi-drug resistance

\section{INTRODUCTION}

Urinary tract infections (UTIs) are the most common infectious disease which are affecting about 150 million cases around the world annually (Flores-Mireles, 2015). UTIs may affect only bladder (cystitis) with localized symptoms (painful urination, urgency and frequency) or may extend to kidneys (pyelonephritis ) with more serious symptoms which can be developed into life threating septicemia (Mahon, 2015). Uropathogenic Escherichia coli (UPEC) is considered the primary cause of the UTI. About $95 \%$ of UTIs are treated empirically (especially uncomplicated cystitis) using several classes of antibiotics such as sulphamethoxazole, fluoro-quinolones and $\beta$-lactams antibiotics (Gupta et al., 2011). 
The increasing resistance rate towards different classes of antibiotics that mainly used in such infections and the emergence of multiple drug resistant (MDR) strains is considered a serious health problem limiting the available treatment options for such infections.

Extended spectrum $\beta$-lactamase (ESBL) is the primary mechanism responsible for $\beta$ - lactams resistance in Enterobacteriaceae where Escherichia coli; especially those involved in UTI; represents the majority of ESBL producing Enterobacteriaceae (Rudresh and Nagarathnamma, 2011). ESBL is plasmid mediated hydrolyzing enzymes being capable of cleavage of extended spectrum oximino-cephalosporin antibiotics and mononbactam but inhibited by $\beta$-lactamase inhibitors especially clavulanic acids (Shaikh et al., 2015). ESBL-producing pathogen also exhibited resistance towards different classes of non $\beta$ - lactams antibiotics which mainly due to plasmid acquisition where ESBL encoding genes enzymes can exist along with other antibiotic resistance genes (Paterson and Bonomo, 2005).

The aim of this study to determine the overall local resistance rate towards different classes of antibiotics and determine the rate of ESBL production among UPEC isolates. Also to determine the correlation between ESBL production and antibiotic resistance among ESBL- producing isolates.

\section{MATERIAL and METHODS}

\section{Bacterial isolates}

A total of 112 UPEC isolates were obtained from patients who were admitted to Urology outpatient clinics of Zagazig University's Hospitals. These patients were suffered from symptoms of urinary tract infections in the period from June 2016 to August 2017.

Among these isolates, 66 UPEC isolates were obtained from patients suffered from cystitis and the remaining 46 isolates were obtained from patients suffered from pyelonephritis

\section{Isolation and identification of UPEC isolates}

All isolates were obtained from clean catch mid-stream urine specimens from patients suffering from symptoms of UTIs. Quantitative urine culture were performed to all urine specimens on nutrient agar and MacConkey agar (Oxoid, Hampshire, UK) using a cut off value $10^{5} \mathrm{CFU} /$ $\mathrm{mL}$ (Wilson and Gaido, 2004). Rose pink colonies with bile precipitation colonies zone on MacConkey agar from positive urine cultures with colony count $\geq 10^{5} \mathrm{CFU} / \mathrm{mL}$ were presumptively identified by microscopical examination and by subculture on Eosin methylene blue agar (Oxoid, Hampshire, UK ). Complete identification was performed using standards biochemical test including Indole production test, methyl red test, vogaus proskaur test, citrate utilization, reaction on Triple sugar iron test and nitrate reduction test (Winn and Koneman, 2006).

All isolates were identified by standard biochemical tests and stored at $-80^{\circ} \mathrm{C}$ as $20 \%$ glycerol stocks.

\section{Antimicrobial susceptibility testing} (AMST)

ASMT was performed according to Clinical and Laboratory Standards Institute guidelines (CLSI, 2015a) using 
standard disk diffusion method. Antimicrobial susceptability was tested against against members belongs to $\beta$ Lactams, fluoro-quinolones, aminoglycosides, tetracycline, folate pathway inhibitors and Nitrofurans antibiotic classes including: Amoxicillinclavulanate (AMC, $20 / 10 \mu \mathrm{g}$ ), Piperacillin-tazobactam (TZP, 100/10 $\mu \mathrm{g})$, ceftriaxone (CRO, $30 \mu \mathrm{g})$, Cefpodoxime (CPD, $10 \mu \mathrm{g})$, Cefuroxime (CXM, $30 \mu \mathrm{g})$, Aztreonam (ATM, $30 \mu \mathrm{g}$ ), Imipenem (IMP, $10 \mu \mathrm{g}$ ), meropenem (MEM, 10 $\mu \mathrm{g}$ ), Gentamicin $(\mathrm{CN}, 10 \mu \mathrm{g})$, Doxycycline (DO, 30 $\mathrm{g}$ ) and Ciprofloxacin (CIP, 5 $\mu \mathrm{g}$ ) which were purchased from Oxoid (Hampshire, UK ). Also antimicrobial susceptability test involved Levofloxacin (LEV, 5 $5 \mathrm{~g}$ ), Ofloxacin (OFX, 5 $\mathrm{g}$ ), Trimethoprimsulfamethoxazole (SXT, 1.25/23.75 $\mu \mathrm{g}$ ) and Nitrofurantoin (F, $300 \mu \mathrm{g})$ which were obtained from Bioanalysis ( Ankra, Turkey).

Colonies from overnight cultures on Tryptone soya agar (TSA) plates (Oxoid, Hampshire, UK) were suspended into saline, the turbidity of the suspension was adjusted to be equivalent to 0.5 McFarland standard. A sterile cotton swab which moistened with the prepared inoculum was used to inoculate the surface of Mueller -Hinton Agar (MHA) plates (Oxoid, Hampshire, UK). Antibiotic discs were applied using fineend forceps and plates incubated at inverted position at $37^{\circ} \mathrm{C}$ for $16-18 \mathrm{hrs}$. Inhibition zone were measured and interpreted as sensitive (S), intermediate (I) or resistant (R) according to CLSI criteria (CLSI, 2015b).

\section{Phenotypic detection of ESBL} production
According to CLSI guidelines, isolates with inhibition zone less than $17 \mathrm{~mm}$ for cefpodoxime, $25 \mathrm{~mm}$ for ceftriaxone and/or $27 \mathrm{~mm}$ for aztreonam are considered as a potential ESBL producing isolates (CLSI, 2015b).

ESBL production among candidate isolates was confirmed by double disk synergy test at standard distance $20 \mathrm{~mm}$ (DDST20) using amoxicillin-clavulanate disk (AMC 20/10 $\mu \mathrm{g}$ ) and three antibiotics representing third-generation cephalosporins include ceftazidime (CTZ, $30 \mu \mathrm{g}$ ), ceftriaxone (CRO, $30 \mu \mathrm{g}$ ) and cefotaxime (CTX, $30 \mu \mathrm{g}$ ) along with cefepime (FEB, $30 \mu \mathrm{g}$ ) as a fourthgeneration cephalosporin. All antibiotic discs which were used in DDST20 were obtained from Oxoid (Hampshire, UK).

Colonies from overnight cultures on TSA plates were suspended into $0.85 \%$ saline, turbidity was adjusted to 0.5 McFarland standard and used to inoculate the surface of (MHA) plates by using a sterile cotton swab. Antibiotic discs representing 3rd and 4th were placed at a distance of $20 \mathrm{~cm}$ center to center from Amoxicillin-clavulanate disc which placed in the center of the plate. Plates were incubated at $37^{\circ} \mathrm{c}$ for $16-18 \mathrm{hrs}$. any enhancement or distortion of the inhibition zone of any antibiotic disc towards amoxicillin-clavulanic acid indicates ESBL production (Garrec et al., 2011).

\section{Statistical analysis}

Statistical analysis was performed using SPSS (version 16.0) program. Comparison of proportions performed using chi-square test, $p$-value $\leq 0.05$ consider statistically significant.

\section{RESULTS}


Zagazig J. Pharm. Sci. June, 2018

Vol. 27, Issue 1, pp. 64- 72

\section{Identification of UPEC isolates}

UPEC isolates were presumptively identified by its growth on MacConkey agar producing rose pink colonies with bile precipitation zone and on Eosin Methylene blue agar by producing black colonies with green metallic sheen. UPEC are Gram negative rods when microscopically identified. These isolates were completely identified by being capable of producing indole, fermentation of sugar and production of acidic product with positive methyl red test, reduce nitrate to nitrite and produce acid with gas on triple sugar iron test. UPEC isolates can't utilize citrate and give false result in vogaus proskaur reaction.

\section{Antimicrobial susceptibility testing}

ISSN 1110-5089

ISSN (on-line) 2356_9786

UPEC isolates exhibited different susceptibility rates towards different antibiotics (Table 1). The highest resistance was observed against doxycycline (98\%) followed by cefuroxime and cefpodoxime $(72 \%$ and $68 \%$ respectively). intermediate resistance was observed against ceftriaxone $(53.5 \%)$ followed by sulphamethoxazole-trimethoprim (54\%), fluoro-quinolones $(48.6 \%-50.5 \%)$ and aztreonam (38\%).

The lower resistance was observed against gentamicin, nitrofurantoin and piperacillin-tazobactam combination (20.5\%, 20.5\% and $15 \%$, respectively). The lowest resistance rate was against imipenem and meropenem (7\% and $7.8 \%$, respectively).

Table 1: Percentage of antibiotic resistance among UPEC isolates

\begin{tabular}{|l|c|}
\hline Antibiotic & $\begin{array}{c}\text { No. (\%) of resistant } \\
\text { isolates }\end{array}$ \\
\hline Doxycycline & $110(98 \%)$ \\
\hline Cefuroxime & $81(72 \%)$ \\
\hline Cefpodoxime & $76(68 \%)$ \\
\hline Ceftriaxone & $60(53.5 \%)$ \\
\hline Sulphamethoxazole-trimethoprim & $61(54 \%)$ \\
\hline Ciprofloxacin & $56(50.5 \%)$ \\
\hline Ofloxacin & $55(49.5 \%)$ \\
\hline Levofloxacin & $54(48.6 \%)$ \\
\hline Aztreonam & $42(38 \%)$ \\
\hline Gentamicin & $23(20.5 \%)$ \\
\hline Nitrofurantoin & $23(20.5 \%)$ \\
\hline Piperacillin-tazobactam & $17(15 \%)$ \\
\hline Imipenem & $8(7 \%)$ \\
\hline Meropenem & $9(7.8 \%)$ \\
\hline
\end{tabular}

\section{ESBL production}

Seventy isolates were considered as potential ESBL producer in the initial antibiotic susceptibility test. Eighty percent of these isolates (56/70) were confirmed to be ESBL producer with overall percentage $50 \%$ of the total isolates. While the remaining proportion remained unconfirmed with overall percentage $12.5 \%$ of the total UPEC isolates (Figure 1). 


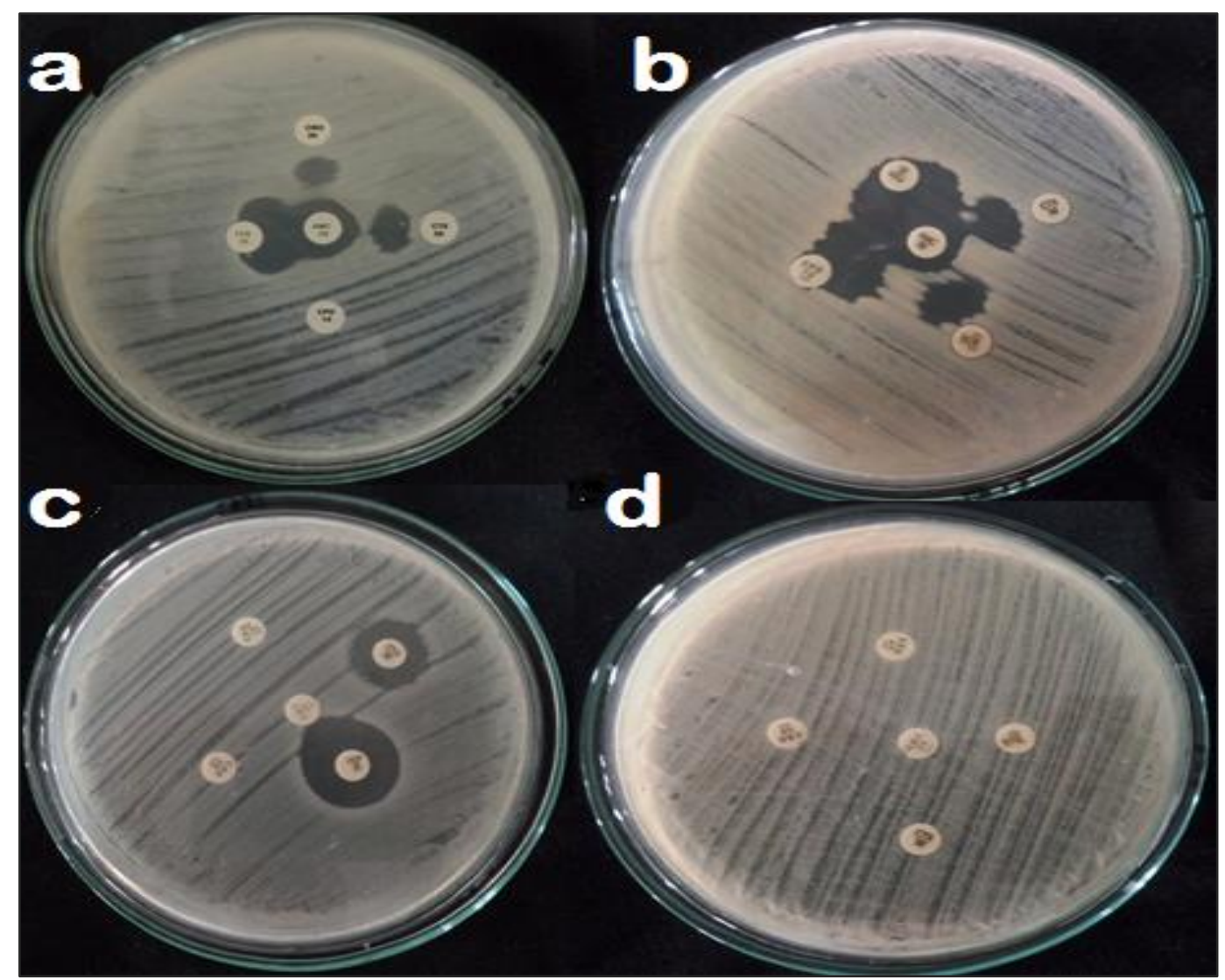

Figure 1: double disk synergy test (DDST): amoxcilin-clavulanic acid disc was placed in the center at $20 \mathrm{~cm}$ center to centre of ceftazidim, cefatriaxone, cefotaxime and cefepime discs. (a) represent synergy of cefepime, ceftazidium and cefotaxime with amoxacilin clavulanic acid disc,(b) represent synergy of four cephalosporin discs with amoxicillin clavulanic disc, (c) represent synergy of cefepime only with amoxicillin clavulanic acid disc and (d) represent no synergy between cephalosporin discs and amoxacilin clavulanic acid disk.

\section{Antibiotic susceptibility in relation to ESBL production:}

ESBL producing UPEC exhibited higher resistance rate towards several antibiotic classes when compared with non-producing isolates

(table. 1). Resistance rate against doxycycline was high in both ESBLproducing and non-producing isolates (99.96\% and 83.8\%, respectively..). ESBL producing isolates exhibited statistically higher resistance rate especially against sulphamethoxazoletrimethoprim and fluoro-quinolones (67.8 $\%$ and $63 \%$, respectively) when compared with non-producing isolates (35.7\% and $19 \%$, respectively). Also, ESBL producing isolates exhibited mild to low resistance towards gentamicin, nitrofurantoin and piperacilintazobactam $(30.3 \%, 17.8 \%$ and $16 \%$, respectively), while non-producing isolates exhibited similar resistance rate towards nitrofurantoin $(9.5 \%)$ and exhibited no resistance towards gentamicin and piperacilin-tazobactam. Carbapenems (meropenem and imipenem) exhibited the lowest resistance rate among ESBL producing isolates. 
Table 2: Comparison the antibiotic resistance among ESBL producing and ESBL nonproducing isolates (a): including only confirmed ESBL producing isolates.

\begin{tabular}{|l|l|l|l|}
\hline Antibiotic & $\begin{array}{l}\text { ESBL producer } \\
(\mathrm{n}=56)\end{array}$ & $\begin{array}{l}\text { ESBL non producer } \\
(\mathrm{n}=42)\end{array}$ & $p$-value \\
\hline Doxycycline & $99.96 \%$ & $83.8 \%$ & 0.002 \\
\hline Sulphamethoxazole-triethoprim & $67.8 \%$ & $35.7 \%$ & 0.0017 \\
\hline Fluoro-quinolone & $63 \%$ & $19 \%$ & $<0.0001$ \\
\hline Gentamicin & $30.3 \%$ & $0 \%$ & $<0.0001$ \\
\hline Nitrofurantoin & $17.8 \%$ & $9.5 \%$ & 0.26 \\
\hline Piperacilin-tazobactam & $16 \%$ & $0 \%$ & 0.006 \\
\hline Imipenem & $1.7 \%$ & $0 \%$ & 0.4 \\
\hline Meropenem & $3.5 \%$ & $0 \%$ & 0.22 \\
\hline
\end{tabular}

\section{DISCUSSION}

Escherichia coli is the most frequent bacterium involved in UTIs including uncomplicated cystitis and pyelonephritis. The problem of empirical treatment besides self-medication and the misusing of antimicrobial agents which present in many countries like ours has a great impact in increasing the local resistance rate with increasing risk of therapy failure (Michael $e t$ al. , 2014).

Several antibiotic classes such as nitrofurantoin, sulphamethoxazoletrimethoprim, fluoro-quinolones are considered appropriate choices for UTIs treatment especially uncomplicated cystitis (Gupta et al., 2011). But the increased local resistance rate towards them especially sulphamethoxazole-trimethoprim and fluoro-quinolones limit their role in the treatment.

Our result demonstrated increased resistance rate towards sulphamethoxazole (54\%) and fluoro-quinolones (48.6\% $50.5 \%$ ) which came in agreement with previous studies (Das et al., 2009; Shariff et al., 2013; Neamati et al., 2015).

Also UPEC exhibited a reduced susceptibility towards $\beta$ - lactams including extenetded spectrum cephalosporins that $50 \%$ of tested isolates were confirmed to be
ESBL-producers. Our results came in agreement with two similar studies performed in Egypt where the first study stated that ESBL production was observed in $54.6 \%$ of UPEC isolates (Morsi and Tash, 2016). And the other study demonstrated that $36 \%$ of UPEC isolates were found to be ESBL producer (Elsayed et al., 2017) confirming the increased rate of ESBL producing UPEC in Egypt.

ESBL production not only restricts the using of $\beta$ lactams antibiotics but also extends to include other classes due to the presence of antibiotic co-resistance among producing strains. Our result demonstrated that the majority of ESBL producing isolates $(85.7 \%)$ exhibited multiple drug resistance nature.

ESBL producing isolates are mainly resistant to doxycycline and exhibited statistically high rate of resistance especially towards sulphamethoxazole-trimethoprim and fluoro-quinolones (67.8\% and 63\%, respectively) that ESBL producing isolates were either being resistance to sulphamethoxazole-trimethoprim or fluoroquinolones along with resistance towards other antibiotics such as gentamicin (30.3\%). Moreover, $47.4 \%$ of ESBL isolates were resistance to both sulphamethoxazoletrimethoprim and fluoro-quinolones. 
This observed high rate antibiotic coresistance among ESBL producing isolates came in agreement with other studies which is appeared to be a world-wide trend with slight variation between countries (Azap et al., 2010; El bouamri et al., 2014; Ali et al., 2016).

However, ESBL producing isolates exhibited high susceptibility towards other antibiotics including carbapenems (imipenem and meropenem) piperacillintazobactam besides nitrofurantoin which can be used in the treatment of these multiple antibiotic resistant ESBL producing UPEC.

Carbapenem consider the drug of choice for the treatment of ESBL producing isolates that on study reported that $>98 \%$ of the ESBL-producing E. coli are susceptible to this antibiotic class (Perez et al.,2007). But the using of carbapenem-sparing agents (with comparable activity) against ESBL producing isolates such as cefepime and fosfomycine is a good option to avoid the emergence of carbapenemase producing strains (Pullukcu et al., 2007; Kim et al., 2018).

\section{Conclusion}

ESBL producing UPEC exhibited multiple antibiotic resistance nature with statistically high resistance rate to other antibiotic classes. This observed antibiotic coexistence is the result of plasmid acquisition carrying ESBL encoding genes along with other antibiotic resistance genes making the available treatment options is limited.

\section{REFERENCES}

Ali, I., Rafaque, Z., Ahmed, S., Malik, S., and Dasti, J. I. (2016). Prevalence of multi-drug resistant uropathogenic Escherichia coli in Potohar region of Pakistan. Asian Pacific Journal of Tropical Biomedicine, 6(1), 60-66.
Azap, Ö. K., Arslan, H., Şerefhanoğlu, K., Çolakoğlu, Ş., Erdoğan, H., Timurkaynak, F., and Senger, $S$. S. (2010). Risk factors for extended-spectrum $\quad \beta$-lactamase positivity in uropathogenic Escherichia coli isolated from community-acquired urinary tract infections. Clinical Microbiology and Infection, 16(2), 147-151.

Clinical and Laboratory Standards Institute (2015a) Performance Standards for Antimicrobial Disk Susceptibility Tests; Approved Standard- Twelifith Edition. CLSI document M02-A11 (ISBN 156238-781-2 [Print]; ISBN 156238-782-0 [Electronic]). Clinical and Laboratory Standards Institute, 950 West Valley Road, Suite 2500, Wayne, Pennsylvania 19087 USA

Clinical and Laboratory Standards Institute. (2015b) Performance standards for antimicrobial susceptibility testing; Twenty-Fifth Informational Supplement. CLSI document M100-S25 (ISBN 156238-989-0 [Print]; ISBN 156238-990-4 [Electronic]). Clinical and Laboratory Standards Institute, 950 West Valley Road, Suite 2500, Wayne, Pennsylvania 19087 USA.

Das, R., Perrelli, E., Towle, V., Van Ness, P. H., and Juthani-Mehta, M. (2009). Antimicrobial susceptibility of bacteria isolated from urine samples obtained from nursing home residents. Infection Control and Hospital Epidemiology: The Official Journal of the Society of Hospital Epidemiologists America, 30(11), 1116-1119.

El bouamri, M. C., Arsalane, L., Kamouni, Y., Yahyaoui, H., Bennouar, N., Berraha, M., and Zouhair, S. (2014). Profil actuel de résistance aux antibiotiques des souches d'Escherichia coli uropathogènes et conséquences 
thérapeutiques. Progrès en Urologie, 24(16), 1058-1062

Elsayed, T. I., Ismail, H. A., Elgamal, S. A., and Gad, A. H. (2017) The occurrence of multidrug resistant E. Coli which produce ESBL and cause urinary tract infections. Journal of Applied Microbiology and Biochemistry. 1, 2-8

Flores-Mireles, A. L., Walker, J. N., Caparon, M., and Hultgren, S. J. (2015). Urinary tract infections: epidemiology, mechanisms of infection and treatment options. Nature Reviews. Microbiology, 13(5), 269-284.

Garrec, H., Drieux-Rouzet, L., Golmard, J. L., Jarlier, V., and Robert, J. (2011). Comparison of nine phenotypic methods for detection of extended-spectrum beta-lactamase production by Enterobacteriaceae. Journal of Clinical Microbiology, 49(3), 1048-1057

Gupta, K., Hooton, T. M., Naber, K. G., Wullt, B., Colgan, R., Miller, L. G., Moran, G. J., Nicolle, L. E., Raz, R., Schaeffer, A. J. and Soper, D. E. (2011). International clinical practice guidelines for the treatment of acute uncomplicated cystitis and pyelonephritis in women: A 2010 update by the infectious diseases society of America and the European society for microbiology and infectious diseases. Clinical Infectious Diseases, 52(5), e103-e120.

Kim, S. A., Altshuler, J., Paris, D., and Fedorenko, M. (2018). Cefepime versus carbapenems for the treatment of urinary tract infections caused by extended-spectrum $\beta$ lactamase-producing

Enterobacteriaceae. International Journal of Antimicrobial Agents, 51(1),155-158.

Mahon, C. R. (2015). Textbook of diagnostic Microbiology, fifth edition: Elsevier Health sciences
Michael, C. A., Dominey-Howes, D., and Labbate, M. (2014). The antimicrobial resistance crisis: causes, consequences and management. Frontiers in Public Health, 2, 145.

Morsi, S. S., and Tash, M. R. (2016). Virulance determinants among Extended-spectrum B-lactamases producers of uropathogenic Eschierchia coli isolates in Zagazig university hospitals, Egypt. Egyptian Journal of Medical Microbiology, 25(2),101-108.

Neamati, F., Firoozeh, F., Saffari, M., and Zibaei, M. (2015). Virulence genes and antimicrobial resistance pattern in uropathogenic Escherichia coli isolated from hospitalized patients in Kashan, Iran. Jundishapur Journal of Microbiology, 8(2), e17514.

Paterson, D. L., and Bonomo, R. A. (2005). Extended-Spectrum $\beta$ Lactamases: a clinical Update. Clinical Microbiology Reviews, 18(4), 657-686.

Perez, F., Endimiani, A., Hujer, K. M., and Bonomo, R. A. (2007). The continuing challenge of ESBLs. Current Opinion in Pharmacology, 7(5), 459-469.

Pullukcu, H., Tasbakan, M., Sipahi, O. R., Yamazhan, T., Aydemir, S., and Ulusoy, S. (2007). Fosfomycin in the treatment of extended spectrum beta-lactamase-producing Escherichia coli-related lower urinary tract infections. International Journal of Antimicrobial Agents, 29(1), 62-65

Rudresh, S. M. and Nagarathnamma, T. (2011). Extended spectrum $\beta$ lactamase producing Enterobacteriaceae \& antibiotic coresistance. The Indian Journal of Medical Research, 133(1), 116-118

Shaikh, S., Fatima, J., Shakil, S., Rizvi, S. M. D., and Kamal, M. A. (2015). Antibiotic resistance and 
extended spectrum beta-lactamases: types, epidemiology and treatment. Saudi Journal of Biological Sciences, 22(1), 90-101.

Shariff V. A. A. R., Shenoy S. and Yadav, T. (2013). The antibiotic susceptibility patterns of uropathogenic Escherichia coli, with special reference to the fluoroquinolones. Journal of Clinical and Diagnostic Research, 7(6), 1027-1030.
Wilson, M. L., and Gaido, L. (2004). Laboratory diagnosis of Urinary tract Infections in adult patients. Clinical Infectious Diseases, 38(8), 1150-1158.

Winn, W. C., and Koneman, E. W. (2006). Koneman's color atlas and textbook of diagnostic microbiology: Lippincott williams and wilikins.

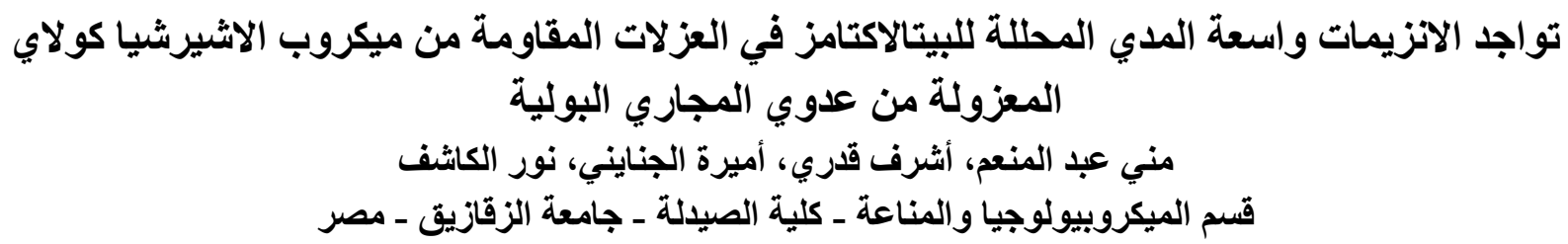

تعد التهابات المساللك البولية من اكثر انواع العدوي البكترية انتشارا التى من الممكن ان تقتصر على المئى الثنانة او تمتد الى الجهاز

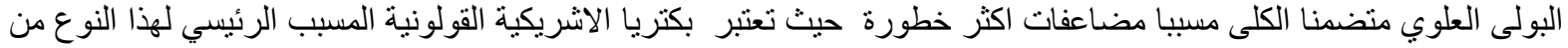
العدوي.

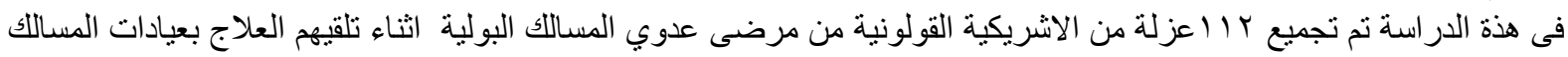

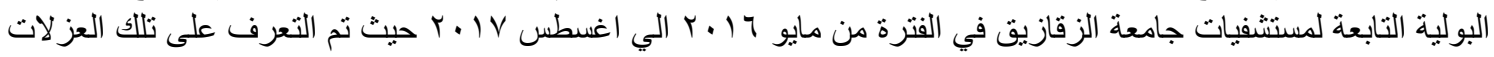

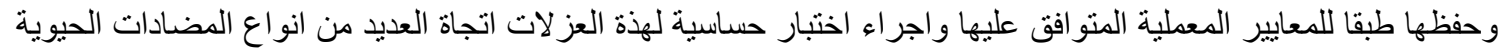

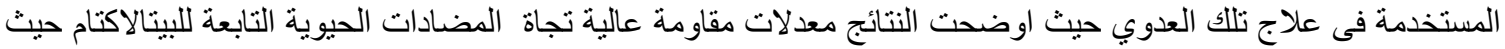

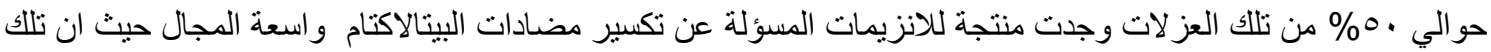

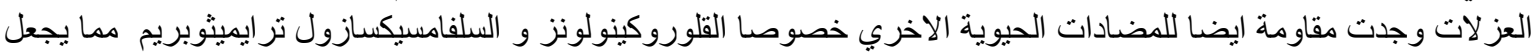

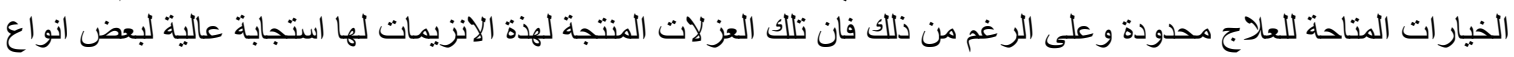

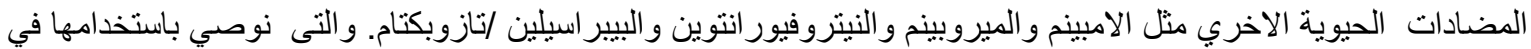
علاج مثل هذة العدوي المسببه بواسطة تللك العزلات ذات التيروبن المقاومة المتعددة. 